\title{
Resonant binding of dielectric particles to metal surface without plasmonics
}

\author{
Evgeny Bulgakov, ${ }^{1,2}$ Konstantin Pichugin, ${ }^{1}$ and Almas Sadreev ${ }^{1}$ \\ ${ }^{1}$ Kirensky Institute of Physics Federal Research Center KSC SB RAS 660036 Krasnoyarsk Russia \\ ${ }^{2}$ Reshetnev Siberian State University of Science and Technology, 660037, Krasnoyarsk, Russia
}

(Dated: March 29, 2021)

\begin{abstract}
High index dielectric spherical particle supports the high- $Q$ resonant Mie modes that results in a regular series of sharp resonances in the radiation pressure. A presence of perfectly conducting metal surface transforms the Mie modes into the extremely high- $Q$ magnetic bonding or electric antibonding modes for close approaching of the sphere to the surface. We show that the electromagnetic plane wave with normal incidence results in repulsive or attractive resonant optical forces relative to metal for excitation of the electric bonding or magnetic anti-bonding resonant modes respectively. A magnitude of resonant optical forces reaches order of one nano Newton of magnitude for micron size of silicon particles and power of light $1 \mathrm{~mW} / \mu \mathrm{m}^{2}$ that exceeds the gravitational force by four orders. However what is the most remarkable there are steady positions for the sphere between pulling and pushing forces that gives rise to resonant binding of the sphere by metal surface. A frequency of mechanical oscillations of particle around the equilibrium positions reaches a magnitude of order $\mathrm{MHz}$.
\end{abstract}

\section{INTRODUCTION}

An illumination of dielectric spherical particle by plane wave with definite wave vector gives rise to radiation pressure which drags the particle along the wave vector because of preservation of momentum. Irvine has shown that regular series of sharp resonances in the radiation pressure superimposes on this placid background of radiation pressure caused by excitation of the Mie resonant modes with high $Q$ factor in dielectric spherical particles [1]. In 1977 Ashkin and Dziedzic reported the first precise observation of these resonances based a force spectroscopy [2]. Illumination of the spherical particles by spatially structured beams can even pull particles because of absence of definite longitudinal momentum [3-5]. However irrespectively there are no equilibrium positions for particles over the axis of beam.

Obviously, a presence of surface which reflects incident plane wave can drastically change a situation with the equilibrium positions for particles, i.e., the surface can trap particles outside. Dielectric surface can trap particles using the evanescent tails of waveguide modes [6] while metal surface can trap particles due to excitation of surface plasmonic waves by oblique plane waves [7-13]. A key issue in particle trapping by evanescent tails is the formation of a stable equilibrium in the transverse direction which is rather close to the surface while in the direction along the surface the stability of particles is remaining in question.

In the present letter we show that a presence of perfectly reflecting metal allows to avoid excitation of surface plasmonic waves and to trap dielectric spheres at certain distances even for normal incidence of plane wave. For the case of perfectly conducting metal the problem of scattering can be solved by resorting to image theory, according to which the field scattered into the accessible half-space by a sphere in the presence of the reflecting surface coincides with the field scattered by the compound object that includes the particle and its image, provided the exciting field is the superposition of the actual incident field and of the field that comes from the image source [14]. Therefore the problem can be considered as a scattering of EM waves by two spheres. A presence of the second particle or any scattering object leads to multiple scattering between the object and particle and can lead to optical binding of particle to the object even under illumination of one beam. This is often referred to peculiar manifestation of optical forces as optical binding, and it was first discovered by Burns et al on a system of two plastic spheres in water in 1989 [15]. Depending on the particle separation, the optical binding leads to attractive or repulsive forces between the particles and, thus, contributes to the formation of stable configurations of particles.

Sharp features in the force spectroscopy, causing mutual attraction or repulsion between successive photonic crystal layers of dielectric spheres under illumination of plane wave has been presented by Antonoyiannakis and Pendry [16]. Each layer is specified by extremely narrow resonances which transform into the bonding and anti-boding resonances for close approaching of the layers. It was revealed that the lower frequency bonding resonance forces push the two layers together and the higher frequency anti-bonding resonance pull them apart. These conclusions are based on an analogy of the Maxwell's equations with the quantum mechanics, in particular with molecular orbitals. Later these disclosures we reported for coupled photonic crystal slabs [17], two planar dielectric photonic metamaterials [18], two silicon spheres [19, 20] and two coaxial disks [21]. Similarly for the case of dielectric sphere with large refractive index at perfectly conducting surface the high- $Q$ Mie resonant modes transform into the bonding TM type or anti-bonding $\mathrm{TE}$ type modes which give rise to attractive or repulsive resonant forces because of boundary conditions. And what is important these forces considerably exceed by a two orders of magnitude the resonant pushing forces in the case of bare sphere $[1,2]$. 

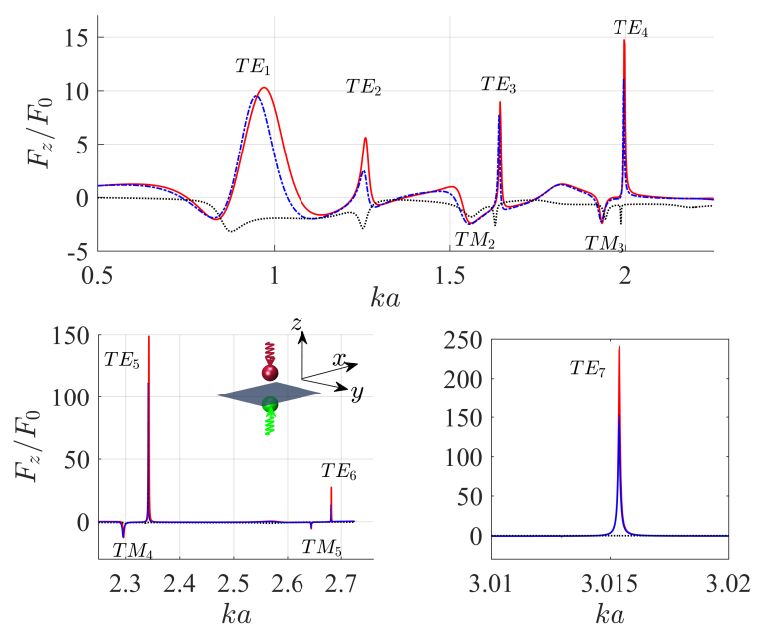

Figure 1. Force normal to silver surface acting on silicon sphere with radius $a=0.55 \mu m$ and refractive index $n=3.464$ at wavelength $\lambda=1.5 \mu \mathrm{m}$ under illumination of plane wave with intensity $P=1 \mathrm{~mW} / \mu \mathrm{m}^{2}$. $F_{0}=8.1 p N$. Black dotted line shows the radiation-pressure acting on bare sphere, red solid line shows the case at distance $L / a=1.2$ from ideal metal, and blue dash-dotted line shows the case of silver metal with refractive index $n=0.14+11 i$ at $\lambda=1.5 \mu m$. Sub indices enumerate orbital momentum $l$ of the Mie resonances of bare sphere. Inset shows sphere (red) positioned at distance $L$ between center of sphere and metal surface and its image (green).

\section{OPTICAL FORCES}

The problem is solved by T-matrix approach [22] that allows us to calculate the optical force acting on the particle via the stress tensor [23]

$$
\begin{gathered}
F_{\alpha}=\int T_{\alpha \beta} d S_{\beta}, \\
T_{\alpha \beta}=\frac{1}{4 \pi} E_{\alpha} E_{\beta}^{*}-\frac{1}{8 \pi} \delta_{\alpha \beta}|\mathbf{E}|^{2}+\frac{1}{4 \pi} H_{\alpha} H_{\beta}^{*}-\frac{1}{8 \pi} \delta_{\alpha \beta}|\mathbf{H}|^{2} .
\end{gathered}
$$

In what follows we consider plane wave incident normally to the metal surface as shown by red wavy line in inset of Fig. 1. That makes the system equivalent to two identical spheres in air in the superposed standing wave $E_{0} \vec{e}_{x} \sin k z$ with even solution for $E_{z}$ and odd for $H_{z}$. Respectively we have odd/even solutions for the tangential components of electric/magnetic fields. The results of calculations are presented in Fig. 1 where dotted line shows the radiation-pressure force onto the bare sphere in air with peaks located at the Mie resonant modes indexed by orbital momentum $l$. That approach differs from consideration of optical forces effected dielectric particles by metal surfaces due to excitation surface plasmon modes by oblique plane waves [8-10, 24].

When two spheres in air are approaching the Mie resonances of each sphere are split and their resonant modes are hybridized forming the bonding and anti-bonding resonant modes irrespective to polarization. Respectively, a sign of optical force between two spheres follows these modes [20]. For the present case of one sphere near metal surface the Mie resonances are not split for approaching of sphere to surface but only shifted as force spectroscopy shows in Fig. 1. The reason is related to that the odd standing wave field $\sin k z$ effecting the sphere and its image can excite only odd resonant modes for tangential components of electric field. One can see that for the TE resonances the shift is positive with repulsive optical forces while for the TM resonances the shift is negative with attractive forces. In order to comprehend this rule we show in Fig. 2 the resonant modes $T E_{5}$ and $T M_{5}$ from the perfectly conducting surface. For the $T E_{5}$ one can see the transverse components of magnetic field dominate compared to the $E_{z}$ in space between sphere and surface. Therefore the stress-tensor component can be approximated as $T_{z z} \approx-\frac{1}{8 \pi}\left(\left|H_{x}\right|^{2}+\left|H_{y}\right|^{2}\right)<0$ to result in positive, i.e., the repulsive force that indeed fully agrees with Fig. 1 for TE resonances. As for as the resonant mode $T M_{5}$ one can see from Fig. 2 that $E_{z}$ dominates over the tangential magnetic field to have $T_{z z} \approx \frac{1}{8 \pi}\left(\left|E_{z}\right|^{2}-\left|H_{x}\right|^{2}-\left|H_{y}\right|^{2}\right)$ positive. Therefore the TM resonances result in attractive force as also agrees with Fig. 1. These results are hold also for the case of real silver metal surface with the refractive index $n=0.14+11 i$ with however slightly reduced values of the optical force as shown in Fig. 1 by blue dash-dotted line.

In Fig. 3 we show results of calculations of optical force versus the frequency of plane wave with normal incidence and power $1 \mathrm{~mW} / \mu \mathrm{m}^{2}$ and distance between sphere and metal surface. Because of oscillating behavior of the optical force $[20,25]$ one can see that attractive resonant forces around the $T M_{l-1}$ resonances is substituted by repulsive 


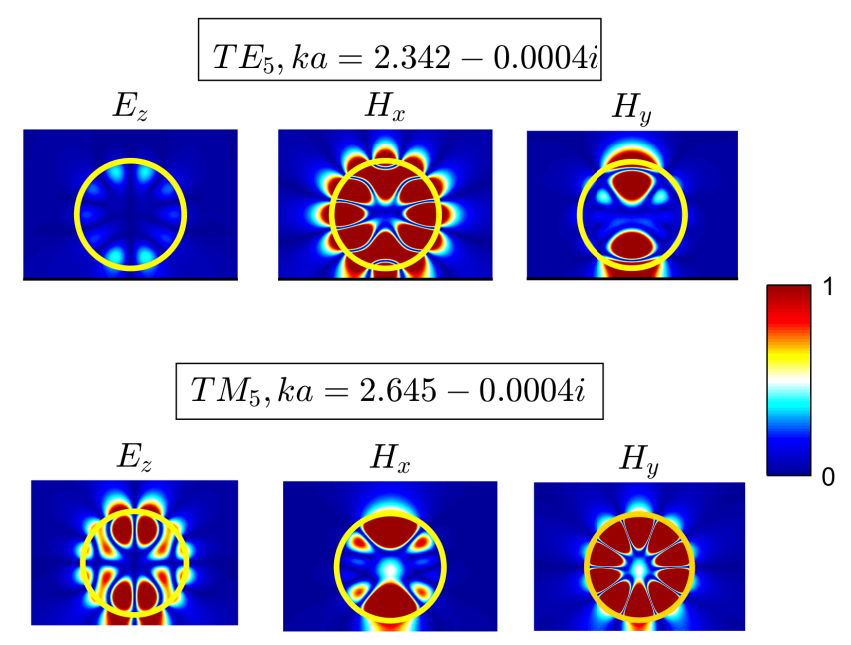

Figure 2. Absolute values of components of EM field of resonant modes at the distance $L=1.2 a$ from ideally conducting surface with corresponding complex resonant frequencies.

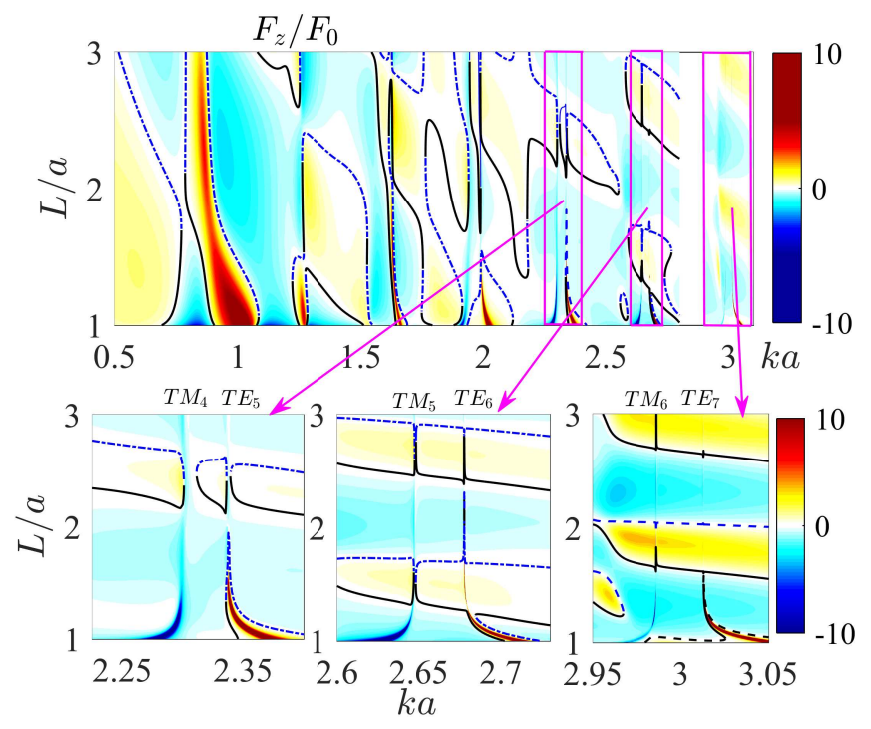

Figure 3. The optical force between sphere and ideally conducting surface vs the frequency and distance for illumination of plane mode with normal incidence. Blue dash solid lines show steady positions of sphere while black solid lines show unsteady positions. $F_{0}=8.1 p N$.

forces around the $T E_{l}$ resonances. Similar to Fig. 1 Fig. 3 manifests force spectroscopy by Ashkin and Dziedzic [2]. Fig. 3 shows also equilibrium positions which depend on distance $L$. What is remarkable there are areas with equilibria of the sphere almost independent of $L$ for high indices $l$. Therefore is to assume that silicon particles of different sizes flow normally to metal surface the particles with definite sizes will bind at definite distances realizing selection by size. That brings new potential to exploit these results for optical trapping and for optical resonant sorting [26].

The insets in Fig. 3 demonstrate considerable enhancement of optical force up to hundreds of pico Newtons that exceed the gravitational forces by four orders for micron size silicon particles. Expectedly, such an enormous enhancement of resonant forces is the result of extremely large $Q$-factors of the resonances as was considered in papers 

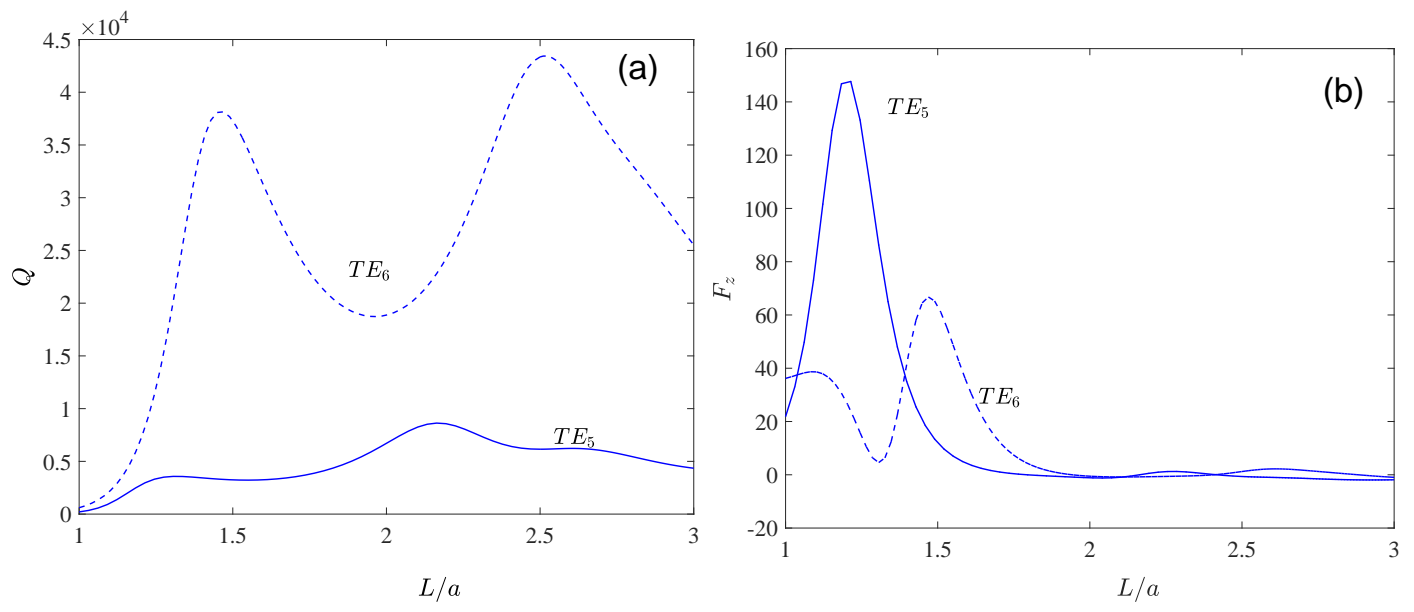

Figure 4. The $Q$ factor (a) and resonant force (b) for the case of excitation of the $T E_{5}$ and $T E_{6}$ resonant modes by plane wave with power $1 \mathrm{~mW} / \mu \mathrm{m}^{2}$ vs the distance between particle and metal silver surface.

$[16,27]$. However Fig. 4 shows that for the present case there is no a strict correlation between the $Q$ factor of resonant mode and the resonant force. Although the $Q$ factor as well as the forces grow with increasing of orbital momentum $l$ as Fig. 1 shows nevertheless we see that the resonant force $T E_{5}$ exceeds the resonant force $T E_{6}$. The reason is related to that not only the $Q$ factor contributes to the resonant optical force but also the coupling constant of incident field with the corresponding resonant mode. In particular, Liu et al have observed already that the higher-Q state exhibits a much weaker coupling to the external radiation [27].

\section{CONCLUSIONS}

One can expect that the larger optical forces will take away the sphere into positions far from the resonance in order to diminish the forces. Indeed, this conclusion holds for the resonances with lower $l$. However, for the extremely high $Q$ resonances with $l=5,6, \ldots$ one can see that the steady distances of silicon sphere from the metal surface are almost independent of $L$ and very close to the resonant frequencies. That tendency for particles to place into positions close to resonances was revealed also in Fabry-Perot resonator with movable mirrors [28]. By differentiation of the force over $z$ it is easy to find vibrational frequency of sphere around equilibrium positions. Fig. 5 shows that the vibrational frequency is around of a few $\mathrm{MHz}$ near steady positions of sphere except a vicinity of resonance $T E_{5}$ where the frequency enhances by one order in magnitude.

Therefore we can conclude that metal surface can strongly bind silicon spherical particles under normal incidence of plane wave with power $1 \mathrm{~mW} / \mu \mathrm{m}^{2}$. However there are two regimes of binding. In the first regime particles are desorbed by metal surface by extremely large optical forces shown in the insets of Fig. 3 by deep blue color. And this process depends on the sizes of particles. In the second regime the particles levitates at the definite distances from the metal surface owing to steady positions shown by blue dash lines in Fig. 3. One can see that these distances of resonant levitation are sensitive to the radius of spheres. Therefore if to assume that the spheres of different sizes are flowing in viscous liquid the plane wave mostly will desorb spheres on surface of metal. However some fracture of particles of definite sizes will be trapped on definite distances from the surface of meta that can pave a way for resonant sorting of particles by size.

\section{Acknowledgements}

The work was supported by Russian Foundation for Basic Research projects No. 19-02-00055 and Russian Scientific Foundation No. 21-12-00131.

[1] W. Irvine, Light scattering by spherical particles: Radiation pressure, asymmetry factor, and extinction cross section, J. Opt. Soc. Am. 55, 16 (1965). 



Figure 5. Optical force (a) and respectively its derivative $\sqrt{\frac{1}{m}\left|\frac{\partial F_{z}}{\partial z}\right|}$ (b) of silicon particle with a mass $m$ around steady positions shown by dash-doted (stable) and solid (unstable) lines.

[2] A. Ashkin and J. M. Dziedzic, Observation of resonances in the radiation pressure on dielectric spheres, Phys. Rev. Lett. 38, 1351 (1977).

[3] A. Novitsky and D. Novitsky, Negative propagation of vector bessel beams, J. Opt. Soc. Am. A 24, 2844 (2007).

[4] J. Chen, J. Ng, Z. Lin, and C. T. Chan, Optical pulling force, Nature Photonics 5, 531 (2011).

[5] H. Li, Y. Cao, L.-M. Zhou, X. Xu, T. Zhu, Y. Shi, C.-W. Qiu, and W. Ding, Optical pulling forces and their applications, Advances in Optics and Photonics 12, 288 (2020).

[6] H. Jaising and O. Hellesø, Radiation forces on a mie particle in the evanescent field of an optical waveguide, Optics Comm. 246, 373 (2005).

[7] G. Volpe, R. Quidant, G. Badenes, and D. Petrov, Surface plasmon radiation forces, Phys. Rev. Lett. 96, 10.1103/physrevlett.96.238101 (2006).

[8] M. Righini, A. Zelenina, C. Girard, and R. Quidant, Parallel and selective trapping in a patterned plasmonic landscape, Nature Physics 3, 477 (2007).

[9] X. Yang, Y. Liu, R. F. Oulton, X. Yin, and X. Zhang, Optical forces in hybrid plasmonic waveguides, Nano Letters 11, 321 (2011).

[10] M. Petrov, S. Sukhov, A. Bogdanov, A. Shalin, and A. Dogariu, Surface plasmon polariton assisted optical pulling force, Laser \& Photonics Reviews 10, 116 (2015).

[11] A. Ivinskaya, M. Petrov, A. Bogdanov, I. Shishkin, P. Ginzburg, and A. Shalin, Plasmon-assisted optical trapping and anti-trapping, Light: Science \& Applications 6, e16258 (2016).

[12] A. V. Maslov, Levitation and propulsion of a mie-resonance particle by a surface plasmon, Opt. Lett. 42, 3327 (2017).

[13] A. Maslov, Binding of resonant dielectric particles to metal surfaces using plasmons, Ann. Phys. 532, 2000129 (2020).

[14] F. Borghese, P. Denti, and R. Saija, Scattering from Model Nonspherical Particles, edited by K. Yasumoto (Springer, 2007).

[15] M. M. Burns, J.-M. Fournier, and J. A. Golovchenko, Optical binding, Phys. Rev. Lett. 63, 1233 (1989).

[16] M. I. Antonoyiannakis and J. B. Pendry, Mie resonances and bonding in photonic crystals, Europhys. Lett. 40, 613 (1997).

[17] V. Liu, M. Povinelli, and S. Fan, Resonance-enhanced optical forces between coupled photonic crystal slabs, Optics Express 17, 21897 (2009).

[18] J. Zhang, K. F. MacDonald, and N. I. Zheludev, Giant optical forces in planar dielectric photonic metamaterials, Opt. Lett. 39, 4883 (2014).

[19] T. Yano, Y. Tsuchimoto, R. P. Zaccaria, A. Toma, A. Portela, and M. Hara, Enhanced optical magnetism for reversed optical binding forces between silicon nanoparticles in the visible region, Optics Express 25, 431 (2017).

[20] E. Bulgakov, K. Pichugin, and A. Sadreev, Giant resonant enhancement of optical binding of dielectric particles, Phys. Rev. A 102, 043518 (2020).

[21] E. N. Bulgakov, K. N. Pichugin, and A. F. Sadreev, Giant resonant enhancement of optical binding of dielectric disks, J. Opt. Soc. Am. B 37, 3335 (2020).

[22] M. I. Mishchenko, J. W. Hovenier, and L. D. T. (editors), Light scattering by nonspherical particles: Theory, measurements, and applications, Measurement Science and Technology 11, 1827 (2000).

[23] M. I. Antonoyiannakis and J. B. Pendry, Electromagnetic forces in photonic crystals, Phys. Rev. B 60, 2363 (1999).

[24] A. V. Maslov, Optical equilibrium for resonant particles induced by surface plasmons of two-dimensional materials, Phys. Rev. B 98, 235414 (2018).

[25] V. Karásek, K. Dholakia, and P. Zemánek, Analysis of optical binding in one dimension, Appl. Phys. B 84, 149 (2006).

[26] D. A. Shilkin, E. Lyubin, M. Shcherbakov, M. Lapine, and A. Fedyanin, Directional optical sorting of silicon nanoparticles, 
ACS Photonics 4, 2312 (2017).

[27] H. Liu, M. Panmai, Y. Peng, and S. Lan, Optical pulling and pushing forces exerted on silicon nanospheres with strong coherent interaction between electric and magnetic resonances, Opt. Express 25, 12357 (2017).

[28] A. F. Sadreev and E. Y. Sherman, Temporal oscillations of light transmission through dielectric microparticles subjected to optically induced motion, Phys. Rev. A 94, 033820 (2016). 